\title{
PARAMETERS ESTIMATION FOR THE SPHERICAL MODEL OF THE HUMAN KNEE JOINT USING VECTOR METHOD
}

\author{
A. CISZKIEWICZ \\ Cracow University of Technology \\ Institute of Machine Design \\ 31-864 Cracow, Al. Jana Pawła II 37, POLAND \\ E-mail: acisz@poczta.fm \\ J. KNAPCZYK \\ State Higher Vocational School in Nowy Sącz \\ 33-300 Nowy Sacz, Staszica 1, POLAND \\ E-mail:j_kn@mech.pk.edu.pl
}

\begin{abstract}
Position and displacement analysis of a spherical model of a human knee joint using the vector method was presented. Sensitivity analysis and parameter estimation were performed using the evolutionary algorithm method. Computer simulations for the mechanism with estimated parameters proved the effectiveness of the prepared software. The method itself can be useful when solving problems concerning the displacement and loads analysis in the knee joint.
\end{abstract}

Key words: model, knee joint, vector method, estimation, position and displacements.

\section{Introduction}

Proper understanding of the human knee joint kinematics can be useful in operation planning of the knee damaged through illness, overuse and in prosthetics. Medical papers on this topic contain mainly descriptions of: the knee joint elements, possible injuries, their diagnosis and treatment. On the other hand, papers concerning biomechanics are mostly focused on the analysis of relative displacements of the femur and the tibia or the loads analysis using one of the appropriate knee models.

Models of the knee joint can be divided into planar and spatial. The simplified, planar model of the knee (Fig.1a) (Stępniewski, 2010) can be analyzed as a mechanism of $3^{\text {rd }}$ class with one linear actuator. Cruciate ligaments $\left(A_{1} B_{1}, A_{2} B_{2}\right)$, connected to the femur by revolute joints $\left(B_{1}, B_{2}\right)$ and to the tibia by revolute joints $\left(A_{1}, A_{2}\right)$, may be considered as rockers of the 4-bar linkage. The drive part of the knee joint consists of the patella $\left(C_{2}\right)$, the patellar tendon $\left(C_{1} C_{2}\right)$ and the muscle $(s)$. Contraction of this muscle generates torque of the femur with respect to the tibia. A planar model of the knee might be sufficient to analyze the displacements and loads occurring during flexion/extension motion of the knee in the sagittal plane, e.g., model of pedaling a bicycle, squatting. However, using a spatial model actual displacements and loads can be analyzed more accurately.

Several variations of the spatial model of the knee joint are presented in Ottoboni et al. (2010). In every model there is: a moving platform corresponding to the femur, a basis corresponding to the tibia and three ligaments (collateral and two cruciate). Ligaments are modeled as fixed-length links connected to the basis and moving platform by spherical joints.

\footnotetext{
* To whom correspondence should be addressed
} 
Respective variants differ in shape of the articular surface of the femur and the tibia. Figure 1c presents the model in which articular surfaces are spherical (indicated by $k_{12}, k_{34}-$ femur and $k_{21}, k_{43}-$ tibia).

a)

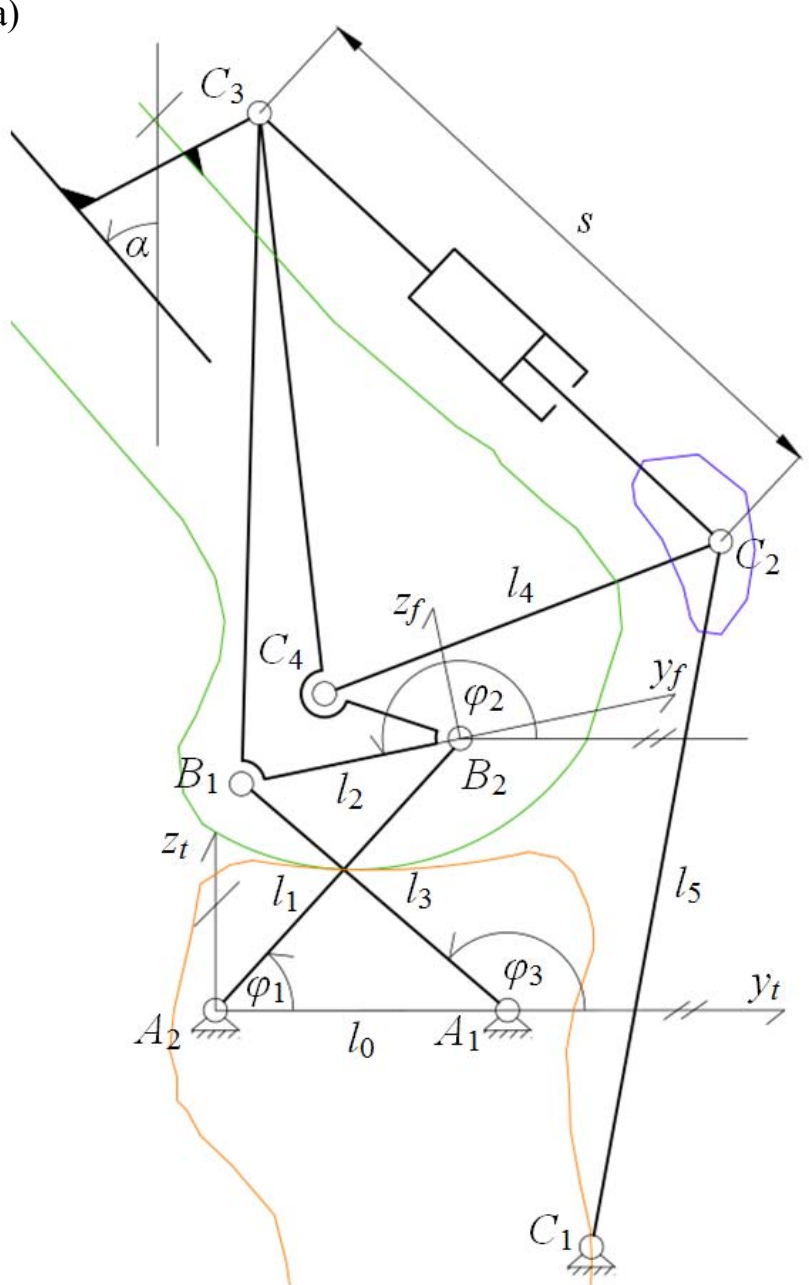

b)

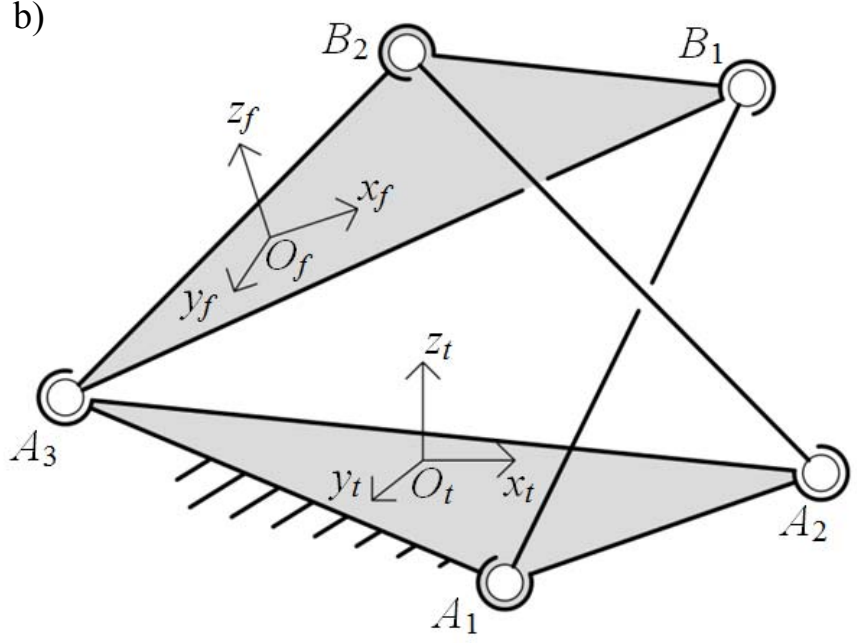




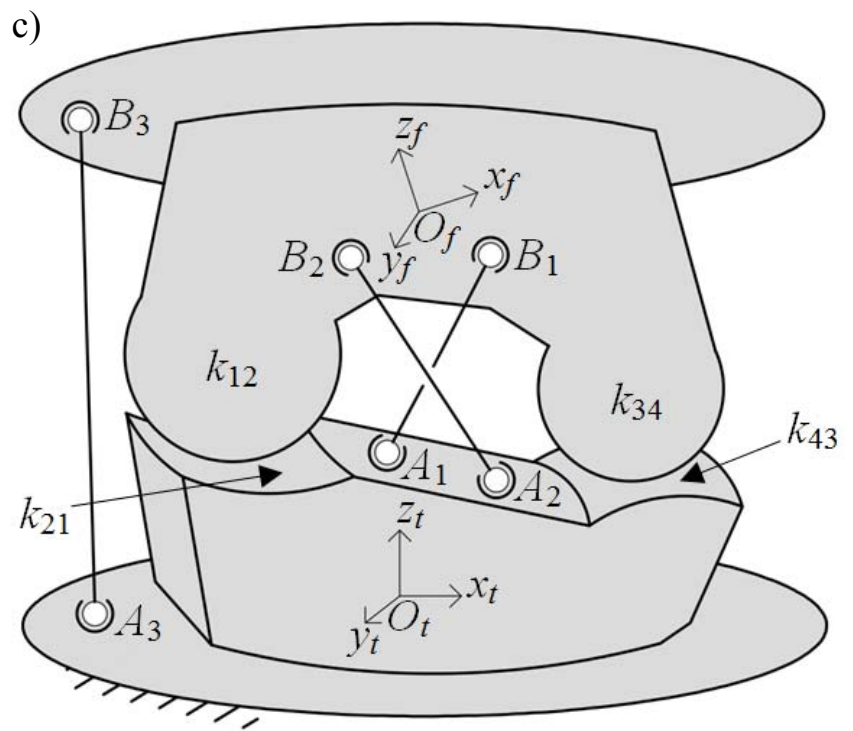

Fig.1. a) Planar model of the knee joint, where: $A_{1} B_{1}, A_{2} B_{2}$ - cruciate ligaments connected to the femur by revolute joints $\left(B_{1}, B_{2}\right)$ and to the tibia by revolute joints $\left(A_{1}, A_{2}\right), C_{2}$ - patella, $C_{1} C_{2}$ - patellar tendon, $s$-substitute muscle. b) Spherical model, where: $A_{1} B_{1}, A_{2} B_{2}$-cruciate ligaments, $A_{3}-$ center of the spherical joint that connects two platforms. c) Spatial model, where: $A_{1} B_{1}, A_{2} B_{2}$ - cruciate ligaments, $A_{3} B_{3}$ - collateral tendon, $k_{12}, k_{34}$ and $k_{21}, k_{43}$ - spherical surfaces. Coordinate systems: $\left\{x_{t} y_{t} z_{t}\right\}$ - tibia reference frame, $\left\{x_{f} y_{f} z_{f}\right\}$ - femur reference frame.

Displacement analysis of the knee joint, presented in Blankevoort et al. (1990), leads to the conclusion that the relative displacement of the femur with respect to the tibia can be described as spherical. A spherical model of the knee joint (Fig.1b) consists of two platforms connected by the spherical joint $\left(A_{3}\right)$ and two fixed-length links ( $A_{1} B_{1}, A_{2} B_{2}$ - cruciate ligaments) connected to platforms by spherical joints. Coordinates of the center of the joint $\left(A_{3}\right)$ that connects two platforms can be computed numerically from the geometrical set of the instantaneous screw axes of the femur with respect to the tibia described in the tibia and in the femur reference frames.

In Ottoboni et al. (2010), Sancisi and Parenti-Castelli (2010) a comparison of the experimental data and results obtained using a spherical and a spatial model (Fig.1c) is presented.

\section{Analysis of the spherical model of the knee joint}

\subsection{Research problem}

Position and displacement analysis of the spherical model of the human knee joint using the constraint equations method (fixed distance between ligaments attachments) leads to a system of 5 nonlinear equations numerically solved in Ottoboni et al. (2010), Sancisi and Parenti-Castelli (2010). In contrast, the vector method (Morecki et al., 2002) yields a closed-form solution dependant on one input variable.

Given two unit vectors $\boldsymbol{e}_{i}^{\boldsymbol{o}}, \boldsymbol{e}_{j}^{\boldsymbol{o}}$ and dot products of those vectors with a third, unknown unit vector, $\boldsymbol{e}_{\boldsymbol{k}}^{\boldsymbol{o}}$ can be determined using the following formula

$$
\boldsymbol{e}_{\boldsymbol{k}}^{\boldsymbol{o}}=\left[\left(c_{i k}-c_{i j} c_{j k}\right) \boldsymbol{e}_{\boldsymbol{i}}^{\boldsymbol{o}}+\left(c_{j k}-c_{i j} c_{i k}\right) \boldsymbol{e}_{\boldsymbol{j}}^{\boldsymbol{o}} \pm \sqrt{D_{k}}\left(\boldsymbol{e}_{\boldsymbol{i}}^{\boldsymbol{o}} \times \boldsymbol{e}_{\boldsymbol{j}}^{\boldsymbol{o}}\right)\right] /\left(1-c_{i j}^{2}\right)
$$

where:

$$
c_{i j}=\boldsymbol{e}_{\boldsymbol{i}}^{\boldsymbol{o}} \cdot \boldsymbol{e}_{\boldsymbol{j}}^{\boldsymbol{o}}, \quad c_{i k}=\boldsymbol{e}_{\boldsymbol{i}}^{\boldsymbol{o}} \cdot \boldsymbol{e}_{\boldsymbol{k}}^{\boldsymbol{o}}, \quad c_{j k}=\boldsymbol{e}_{\boldsymbol{j}}^{\boldsymbol{o}} \cdot \boldsymbol{e}_{\boldsymbol{k}}^{\boldsymbol{o}}, \quad D_{k}=1-c_{i j}^{2}-c_{i k}^{2}-c_{j k}^{2}+2 c_{i j} c_{i k} c_{j k} .
$$


In equations mentioned below the following nomenclature is used:

$\boldsymbol{a}_{\boldsymbol{i}} \quad$ - position vector of point $A_{i}^{t}$ with respect to point $O_{t}$,

$\boldsymbol{a}_{i j} \quad-$ vector from point $A_{i}^{t}$ to point $A_{j}^{t}$,

$\boldsymbol{b}_{\boldsymbol{i}} \quad$ - position vector of point $B_{i}^{t}$ with respect to point $O_{t}$,

$\boldsymbol{b}_{i j} \quad-$ vector from point $B_{i}^{t}$ to point $B_{j}^{t}$,

$\boldsymbol{d}_{i j} \quad-$ vector from point $B_{i}^{t}\left(A_{i}^{t}\right)$ to point $A_{j}^{t}\left(B_{j}^{t}\right)$.
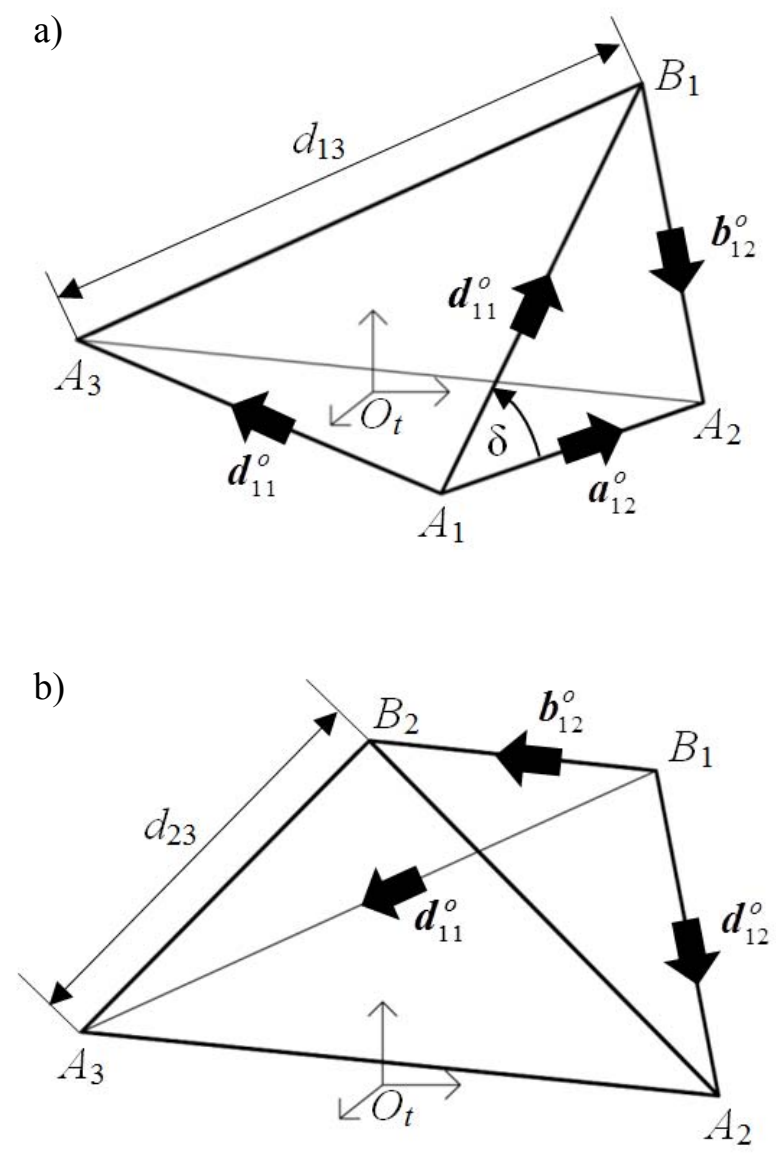

Fig.2. a) First tetrahedron (at basis). b) Second tetrahedron (at moving platform).

The spherical mechanism of the knee joint can be divided into two tetrahedrons (Fig.2). In the first tetrahedron (Fig.2a) only the unit vector $\boldsymbol{d}_{11}^{o}$ that determines the axis of the first cruciate ligament is unknown. The Dot product of the unknown vector $\boldsymbol{d}_{11}^{o}$ with the unit vector $\boldsymbol{a}_{13}^{o}$ can be written using a cosine formula

$$
\boldsymbol{a}_{13}^{o} \cdot \boldsymbol{d}_{11}^{o}=\frac{d_{11}^{2}+a_{13}^{2}-d_{13}^{2}}{2 d_{11} a_{13}}
$$


The Dot product of unit vectors $\boldsymbol{a}_{12}^{o}$ and $\boldsymbol{d}_{11}^{o}$ is the input variable in the model and is denoted as follows

$$
\boldsymbol{a}_{12}^{o} \cdot \boldsymbol{d}_{11}^{o}=\cos \delta
$$

The unit vector $\boldsymbol{d}_{11}^{o}$ is obtained using Eq.(2.1). It is important that $\boldsymbol{d}_{11}^{o}$ can define two different directions depending on the sign before the square root.

Given the unit vector $\boldsymbol{d}_{11}^{o}$ the vector $\boldsymbol{d}_{12}$ can be calculated

$$
\boldsymbol{d}_{12}=\boldsymbol{a}_{12}-\boldsymbol{d}_{11}^{o} d_{11}
$$

Coordinates of point $B_{l}^{t}$ are now known and can be computed using the following formula

$$
\boldsymbol{b}_{1}=\boldsymbol{a}_{1}+\boldsymbol{d}_{11}^{o} d_{11}
$$

In the second tetrahedron (Fig.2b) the unit vector $\boldsymbol{b}_{12}^{o}$ is unknown. In order to find it, unit vectors $\boldsymbol{d}_{12}^{o}, \boldsymbol{d}_{13}^{o}$ and their dot products with $\boldsymbol{b}_{12}^{o}$ are required. The unit vector $\boldsymbol{d}_{12}^{o}$ can be obtained by dividing the vector $\boldsymbol{d}_{12}$ by its length. The Dot product of vectors $\boldsymbol{b}_{12}^{o}$ and $\boldsymbol{d}_{12}^{o}$ can be written using a cosine formula

$$
\boldsymbol{b}_{12}^{o} \cdot \boldsymbol{d}_{12}^{o}=\frac{b_{12}^{2}+d_{12}^{2}-d_{22}^{2}}{2 b_{12} d_{12}}
$$

It is worth noting that vector $\boldsymbol{d}_{13}$ can be obtained using the following formula

$$
\boldsymbol{d}_{13}=\boldsymbol{a}_{3}-\boldsymbol{b}_{1}
$$

The only unknown that remains is the dot product of vectors $\boldsymbol{d}_{13}^{o}$ and $\boldsymbol{b}_{12}^{o}$. In this case it is also possible to find it using cosine formula

$$
\boldsymbol{b}_{12}^{o} \cdot \boldsymbol{d}_{13}^{o}=\frac{b_{12}^{2}+d_{13}^{2}-d_{23}^{2}}{2 b_{12} d_{13}}
$$

Lastly, formula (2.1) can be used to obtain the unit vector $\boldsymbol{b}_{12}^{o}$

$$
\boldsymbol{b}_{2}=\boldsymbol{b}_{1}+\boldsymbol{b}_{12}^{o} b_{12}
$$

Thus, the coordinates of points $B_{1}^{t}$ and $B_{2}^{t}$ in the tibia reference frame are obtained. It is worth mentioning that to compute the position and rotation of the moving platform (femur) with respect to the basis (tibia) only one input variable is required - angle $\delta$.

Furthermore, the second cruciate ligament $\left(d_{22}\right)$ can be used in the displacements analysis of the spherical mechanism. However, such approach yields limited results. 


\subsection{Computing linear and angular displacements}

Position and displacement analysis of the spherical model for the knee joint using the vector method gives the solution in the form of position vectors of three points located on the femur and described in the tibia reference frame $\left\{x_{t} y_{t} z_{t}\right\}$. For the known coordinates of these vectors it is possible to find the rotation matrix and translation vector of the femur reference frame with respect to the tibia by using Horn's closedform solution of absolute orientation described by unit quaternions and presented in Horn (1987).

Sets of the coordinates of vectors $\boldsymbol{b}_{\boldsymbol{i}}(i=1 \ldots n, n \geq 3)$ that define the positions of points $B_{i}$ in two reference frames $\left\{x_{t} y_{t} z_{t}\right\}$ and $\left\{x_{f} y_{f} z_{f}\right\}$ are used to find the position vector of the centroid in respective reference frames

$$
\boldsymbol{b}_{c}=\frac{1}{n} \sum_{i=1}^{n} \boldsymbol{b}_{i}, \quad \boldsymbol{b}_{c}^{f}=\frac{1}{n} \sum_{i=1}^{n} \boldsymbol{b}_{i}^{f}
$$

If the coordinates of the centroid are subtracted from the coordinates of the given points, then experimental data can be expressed with respect to the centroid (in respective reference frames)

$$
\boldsymbol{b}_{i c}=\boldsymbol{b}_{i}-\boldsymbol{b}_{c}, \quad \boldsymbol{b}_{i c}^{f}=\boldsymbol{b}_{i}^{f}-\boldsymbol{b}_{c}^{f} .
$$

Then the values of nine variables $S_{x x}, S_{x y}, \ldots, S_{z z}$ are computed as follows

$$
S_{x x}=\sum_{i=1}^{n} b_{i c x}^{f} b_{i c x}, \quad S_{x y}=\sum_{i=1}^{n} b_{i c x}^{f} b_{i c y}, \quad \ldots
$$

and so on. In the next step a unit quaternion is calculated. This quaternion defines the axis of rotation and the angle of rotation around this axis. In order to find it the following matrix is required

$$
\boldsymbol{N}=\left[\begin{array}{llll}
a & e & h & j \\
e & b & f & i \\
h & f & c & g \\
j & i & g & d
\end{array}\right],
$$

where: $a=S_{x x}+S_{y y}+S_{z z}, \quad b=S_{x x}-S_{y y}-S_{z z}, \quad c=-S_{x x}+S_{y y}-S_{z z}, \quad d=-S_{x x}-S_{y y}+S_{z z}, \quad e=S_{y z}-S_{z y}$, $f=S_{x y}+S_{y x}, g=S_{y z}+S_{z y}, h=S_{z x}-S_{x z}, i=S_{z x}+S_{x z}, j=S_{x y}-S_{y x}$.

The unit quaternion $\boldsymbol{q}=q_{0}+q_{x} \boldsymbol{i}+q_{y} \boldsymbol{j}+q_{z} \boldsymbol{k}$ is obtained as an eigenvector corresponding to the largest eigenvalue of $\boldsymbol{N}$. After this, the rotation matrix can be calculated as follows

$$
\boldsymbol{R}=\left[\begin{array}{lll}
r_{11} & r_{12} & r_{13} \\
r_{21} & r_{22} & r_{23} \\
r_{31} & r_{23} & r_{33}
\end{array}\right],
$$

where 


$$
\begin{array}{lll}
r_{11}=q_{0}^{2}+q_{x}^{2}-q_{y}^{2}-q_{z}^{2}, & r_{12}=2\left(q_{x} q_{y}-q_{0} q_{z}\right), & r_{13}=2\left(q_{x} q_{z}+q_{0} q_{y}\right), \\
r_{21}=2\left(q_{y} q_{x}+q_{0} q_{z}\right), & r_{22}=q_{0}^{2}-q_{x}^{2}+q_{y}^{2}-q_{z}^{2}, & r_{23}=2\left(q_{y} q_{z}-q_{0} q_{x}\right), \\
r_{31}=2\left(q_{z} q_{x}-q_{0} q_{y}\right), & r_{32}=2\left(q_{z} q_{y}+q_{0} q_{x}\right), & r_{33}=q_{0}^{2}-q_{x}^{2}-q_{y}^{2}+q_{z}^{2} .
\end{array}
$$

The translation vector $\boldsymbol{p}$ can be obtained as a difference between two position vectors: position of the centroid described in the tibia reference frame and the same point described in the rotated femur reference frame

$$
\boldsymbol{p}=\boldsymbol{b}_{\boldsymbol{c}}-\boldsymbol{R} \boldsymbol{b}_{c}^{f}
$$

The sequence of rotations is assumed as per (Ottoboni et al., 2010; Sancisi and Parenti-Castelli, 2010) and presented earlier in Grood and Suntay (1983)

$$
R=\left[\begin{array}{ccc}
c \alpha c \gamma+s \alpha s \beta s \gamma & -s \alpha c \gamma+c \alpha s \beta s \gamma & -c \beta s \gamma \\
s \alpha c \beta & c \alpha c \beta & s \beta \\
c \alpha s \gamma-s \alpha s \beta c \gamma & -s \alpha s \gamma-c \alpha s \beta c \gamma & c \beta c \gamma
\end{array}\right],
$$

where $s \alpha=\sin \alpha, c \alpha=\cos \alpha$.

Values of individual angles can be calculated using the following formulas

$$
\begin{aligned}
& \beta=a \tan 2\left(r_{23}, \pm \sqrt{1-r_{23}^{2}}\right), \\
& \alpha=a \tan 2\left(\frac{r_{21}}{\cos \beta}, \frac{r_{22}}{\cos \beta}\right), \\
& \gamma=a \tan 2\left(\frac{-r_{13}}{\cos \beta}, \frac{r_{33}}{\cos \beta}\right) .
\end{aligned}
$$

In medical terminology the following description is used: $\alpha-$ flexion $(+) /$ extension(-), $\beta-\operatorname{abduction}(-) /$ adduction $(+), \gamma-\operatorname{external}(-) /$ internal $(+)$ rotation.

\subsection{Coordinates of the center of the spherical joint that connects the platforms}

It is worth noting that the center of the spherical joint that connects two platforms is a virtual point. Coordinates of this point can be numerically computed using a set of several positions of the femur and tibia as parts of the knee joint. Positions of these parts can be determined from coordinate measurements of the points located on the studied knee.

In order to find the coordinates of the spherical joint center, the mechanism dimensions and the set of several positions of the knee joint parts are required. Given two different positions of a rigid body, screw parameters of this body displacement can be computed. Knowing the number of the body positions it is 
possible to calculate a geometric set of the instantaneous screw axes. In this article the method based on Rodrigues equation (Fenton and Shi, 1990) is used to compute the screw parameters.

Coordinates of the joint $A_{3}$ in the femur and the tibia reference frames can be obtained numerically (Ottoboni et al., 2010). Evolutionary algorithm is used to find the point $A_{3}$ with minimum of $g$

$$
g=\sqrt{\sum_{j=1}^{n} l_{j}^{2}},
$$

where: $l_{j}$ - distance of the selected point from the $j$ instantaneous screw axis, $n$ - number of instantaneous screw axes.

A great advantage of the evolutionary algorithm is that there is no need for the starting solution. One of its drawbacks is high computational complexity that greatly depends on the number of instantaneous screw axes.

\subsection{Data preparation}

Input data set for the displacement analysis contains vectors: $\boldsymbol{b}_{i}^{f}(i=1,2)-$ vector positions of points $B_{i}, \boldsymbol{a}_{3}^{f}$ - vector position of point $A_{3}$ in the femur reference frame, $\boldsymbol{a}_{i}(i=1,2,3)$ defining positions of points $A_{i}$ in the tibia reference frame and ligaments lengths $d_{11}$ and $d_{22}$. The assumed 20 parameters ligaments lengths and coordinates of points in $[\mathrm{mm}]$ - are listed below

$$
\begin{aligned}
& d_{11}=36.6, \quad d_{22}=44.3, \\
& \boldsymbol{a}_{1}=\left[\begin{array}{c}
17.8 \\
-3.8 \\
-0.7
\end{array}\right], \quad \boldsymbol{a}_{2}=\left[\begin{array}{c}
-23.9 \\
-15.3 \\
-7.9
\end{array}\right], \quad \boldsymbol{a}_{3}=\left[\begin{array}{c}
-3.6 \\
17.0 \\
-9.3
\end{array}\right], \\
& \boldsymbol{b}_{1}^{f}=\left[\begin{array}{c}
-6.4 \\
4.8 \\
6.5
\end{array}\right], \quad \boldsymbol{b}_{2}^{f}=\left[\begin{array}{c}
1.8 \\
-2.3 \\
0.2
\end{array}\right], \quad \boldsymbol{a}_{3}^{f}=\left[\begin{array}{c}
-5.6 \\
-0.1 \\
-7.0
\end{array}\right] \text {. }
\end{aligned}
$$

Vectors $\boldsymbol{a}_{1}, \boldsymbol{a}_{2}, \boldsymbol{b}_{1}^{f}$ and $\boldsymbol{b}_{2}^{f}$ are assumed as per (Sancisi and Parenti-Castelli, 2008). Vectors $\boldsymbol{a}_{3}$ and $\boldsymbol{a}_{3}^{f}$ were computed from the geometrical set of the instantaneous screw axes using the aforementioned procedure. The set of positions of the knee joint parts, needed to compute $\boldsymbol{a}_{3}$ and $\boldsymbol{a}_{3}^{f}$, was assumed as per (Sancisi and Parenti-Castelli, 2008).

\subsection{Numerical example}

A numerical example of the transformation matrix calculated at $\delta=60^{\circ}$ is presented below. The input data set was mentioned before. Using formulas (2.2)-(2.9) vectors $\boldsymbol{b}_{1}$ and $\boldsymbol{b}_{2}$ were calculated. After this, coordinates of the centroid were computed using Eqs (2.10) (measured in $[\mathrm{mm}]$ ) 


$$
\boldsymbol{b}_{1}=\left[\begin{array}{c}
-9.0 \\
20.8 \\
3.5
\end{array}\right], \quad \boldsymbol{b}_{2}=\left[\begin{array}{c}
2.6 \\
19.5 \\
-1.2
\end{array}\right], \quad \boldsymbol{b}_{\boldsymbol{c}}=\left[\begin{array}{c}
-3.3 \\
19.1 \\
-2.3
\end{array}\right], \quad \boldsymbol{b}_{c}^{f}=\left[\begin{array}{c}
-3.4 \\
0.8 \\
-0.1
\end{array}\right]
$$

In the next step the point coordinates with respect to the centroid were obtained using Eqs (2.11). Then, the values of the nine variables $S_{x x}, S_{x y}, \ldots, S_{z z}$ were calculated using Eqs (2.12). Finally, the elements $a, b, \ldots, i$ of the matrix $\boldsymbol{N}$ were computed using Eq.(2.13) (in $\left[\mathrm{mm}^{2}\right]$ )

$$
\boldsymbol{N}=\left[\begin{array}{cccc}
142.8 & 0.3 & -37.5 & 42.4 \\
0.3 & -45.9 & -39.2 & -30.1 \\
-37.5 & -39.2 & -127.9 & 51.9 \\
42.4 & -30.1 & 51.9 & 31.0
\end{array}\right]
$$

Eigenvalues and eigenvectors of the aforementioned matrix were found with the help of the mathematical software (e.g., MATLAB). The resulting unit quaternion that corresponds to the largest eigenvalue of $N$ is written below

$$
\boldsymbol{q}=0.9524-0.0293 \boldsymbol{i}-0.0670 \boldsymbol{j}+0.2961 \boldsymbol{k} .
$$

Given the unit quaternion $\boldsymbol{q}$ the rotation matrix and translation vector were calculated using Eqs (2.15) and (2.16)

$$
\boldsymbol{R}=\left[\begin{array}{ccc}
0.8157 & -0.5601 & -0.1450 \\
0.5679 & 0.8229 & 0.0162 \\
0.1102 & -0.0956 & 0.9893
\end{array}\right], \quad \boldsymbol{p}=\left[\begin{array}{c}
-0.1 \\
20.4 \\
-1.7
\end{array}\right] \mathrm{mm}
$$

\subsection{Sensitivity analysis}

Sensitivity analysis enables the user to select the geometrical parameters of the model that have the greatest influence on the results.

In order to study the sensitivity of the model the value of the examined parameter was modified by $\pm 1 \mathrm{~mm}$. Then, its influence on the results was computed as a sum of indicators $\Delta \beta, \Delta \gamma, \Delta p_{x}, \Delta p_{y}, \Delta p_{z}$. Each indicator can be calculated as per (Sancisi and Parenti-Castelli, 2008)

$$
\Delta x=\frac{1}{n z_{x}^{2}} \sum_{i=1}^{n}\left[x_{s}\left(\alpha_{i}\right)-x_{r}\left(\alpha_{i}\right)\right]^{2}
$$

where: $\Delta x$ - indicator of $x \in\left\{\beta, \gamma, p_{x}, p_{y}, p_{z}\right\}, x_{s}\left(\alpha_{i}\right)$ - value of $x$ obtained using the spherical model at $\alpha_{i}$ (here: $\left.\alpha_{i} \in\left\langle 0^{\circ} ; 80^{\circ}\right\rangle, \Delta \alpha_{i}=10^{\circ}\right), x_{r}\left(\alpha_{i}\right)$ - measured value of $x$ at $\alpha_{i}, z_{x}$ - range of $x$ obtained from experimental data, $n$ - number of the flexion angle set elements (here: $n=9$ ). The influence of the examined parameter can be computed as follows 


$$
\sigma=\left(w_{1} \Delta \beta+w_{2} \Delta \gamma+w_{3} \Delta p_{x}+w_{4} \Delta p_{y}+w_{5} \Delta p_{z}\right) \cdot 100 \%
$$

where: $w_{i}$ - weight factor of the respective indicator (here: $w_{i}=1(i=1 . .5)$ ).

The range of the flexion angle is limited because out of the assumed range the real solution of the spherical model did not occur (using initial data set).

Table 1 . Sensitivity analysis - selected results.

\begin{tabular}{c|c|c|c|c|c|c} 
Linear parameter & $d_{22}$ & $b_{2 x}^{f}$ & $a_{3 y}^{f}$ & $a_{2 y}^{f}$ & $\ldots$ & $a_{1 z}^{t}$ \\
\hline$\sigma[\%]$ & 42.4 & 30.3 & 29.0 & 26.5 & $\ldots$ & 0.4
\end{tabular}

The cruciate ligament length $d_{22}$ and parameters $b_{2 x}^{f}, a_{3 y}^{f}$ have the greatest influence on the results. On the contrary, parameters $a_{1 z}^{t}, b_{1 z}^{f}$ are negligibly influential. Sensitivity analysis allows the exclusion of two geometrical parameters from the estimation procedure and the numerical complexity of this task is reduced.

\subsection{Parameters estimation}

Parameters estimation was implemented using an evolutionary algorithm. The evolutionary algorithm was chosen because of ease of rejecting unsatisfactory solutions (e.g., flexion angle's range too narrow, no real solution, real solution out of the knee range of motion).

A comparison of the results obtained from the spherical model and experimental data is performed using the aforementioned relative indicators calculated using Eq.(2.21). Hence, the objective function can be written as follows

$$
f=\left(w_{1} \Delta \beta+w_{2} \Delta \gamma+w_{3} \Delta p_{x}+w_{4} \Delta p_{y}+w_{5} \Delta p_{z}\right)+r
$$

where: $w_{i}$ - weight factor of the respective indicator (here: $\left.w_{i}=1(i=1 . .5)\right), r$-penalty function (assumes a large value if for the selected dataset there is no real solution or the range of the flexion angle is unsatisfactory). It is worth mentioning that parameters estimation is now a single-objective, bounded optimization problem.

The working principle of the evolutionary algorithm is exemplified on the flow chart presented in Fig.3. Note that a group of specimens is called population and an individual specimen is a solution (set of geometrical parameters). The initial population is drawn (although values of the geometrical parameters are bounded). Then the evolutionary algorithm uses the principles of evolution, through appropriately designed genetic operators (selection, crossover, mutation), to generate new populations using the initial population as the basis. The procedure stops if there is no improvement of solution, generations limit or time limit has been reached. 


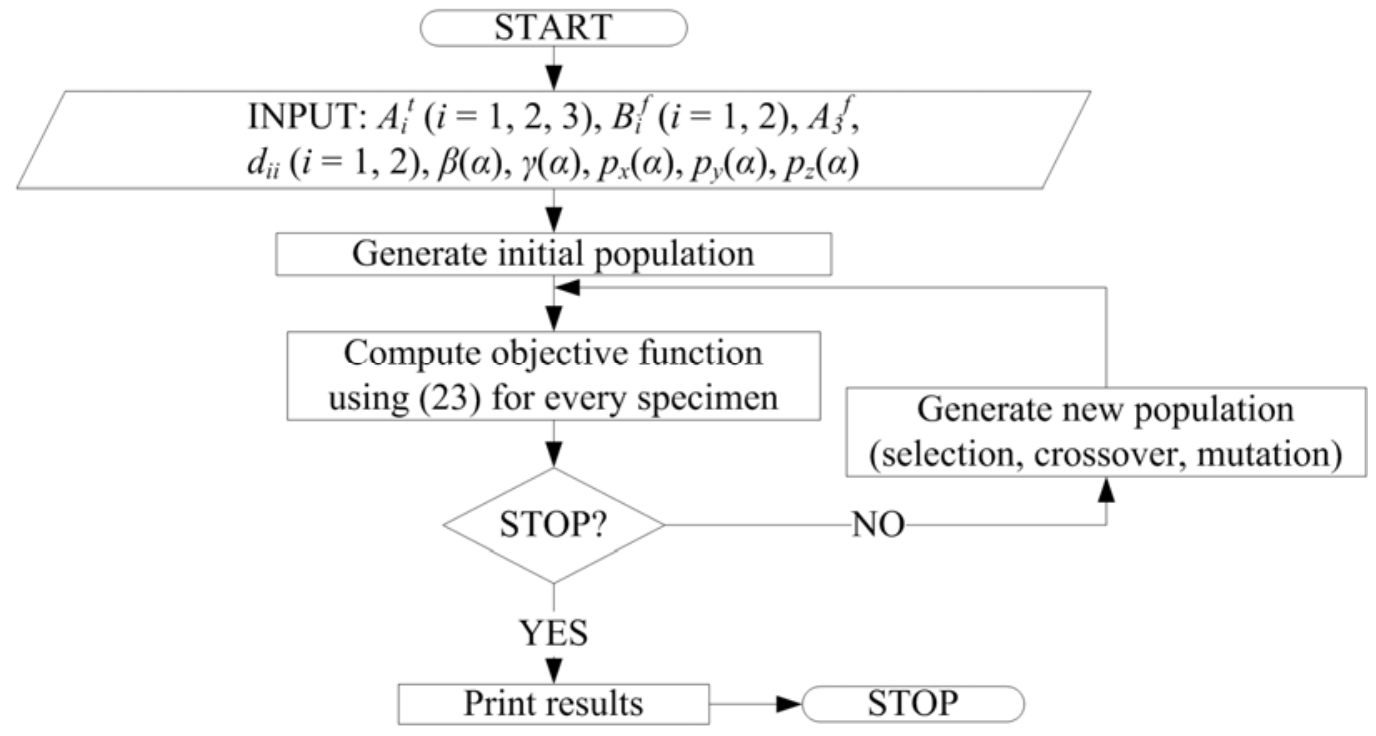

Fig.3. Flow chart - parameters estimation procedure.

\section{Results}

Parameters estimation was performed twice - first for the model with 20 geometrical parameters and then for the model with 18 geometrical parameters (selected with sensitivity analysis). Such an approach enables the evaluation of the validity of sensitivity analysis. Results of the parameters estimation for the model with 20 parameters are as follows (points coordinates and ligaments lengths in $[\mathrm{mm}]$ )

$$
\begin{aligned}
& d_{11}=38.8, \quad d_{22}=45.6, \\
& \boldsymbol{a}_{1}=\left[\begin{array}{c}
16.1 \\
-5.8 \\
-0.1
\end{array}\right], \quad \boldsymbol{a}_{2}=\left[\begin{array}{c}
-23.1 \\
-18.0 \\
-4.9
\end{array}\right], \quad \boldsymbol{a}_{3}=\left[\begin{array}{c}
-3.0 \\
20.0 \\
-10.0
\end{array}\right], \\
& \boldsymbol{b}_{1}^{f}=\left[\begin{array}{c}
-3.9 \\
6.0 \\
8.8
\end{array}\right], \quad \boldsymbol{b}_{2}^{f}=\left[\begin{array}{l}
0.0 \\
0.1 \\
3.2
\end{array}\right], \quad \boldsymbol{a}_{3}^{f}=\left[\begin{array}{c}
-3.1 \\
0.4 \\
-6.9
\end{array}\right]
\end{aligned}
$$

Summarized results of the parameters estimation of the model with 18 parameters and 2 constant coordinates (selected with sensitivity analysis: $a_{1 z}^{t}, b_{1 z}^{f}$ ) are presented below (coordinates and ligaments lengths in $[\mathrm{mm}]$ )

$$
\begin{aligned}
d_{11}=36.7, & d_{22}=44.6, \\
\boldsymbol{a}_{1}=\left[\begin{array}{c}
16.7 \\
-2.3 \\
-0.7
\end{array}\right], & \boldsymbol{a}_{2}=\left[\begin{array}{c}
-22.5 \\
-16.8 \\
-6.1
\end{array}\right], \quad \boldsymbol{a}_{3}=\left[\begin{array}{c}
-2.5 \\
18.8 \\
-10.8
\end{array}\right],
\end{aligned}
$$




$$
\boldsymbol{b}_{1}^{f}=\left[\begin{array}{c}
-6.1 \\
6.8 \\
6.5
\end{array}\right], \quad \boldsymbol{b}_{2}^{f}=\left[\begin{array}{c}
0.2 \\
-1.1 \\
2.2
\end{array}\right], \quad \boldsymbol{a}_{3}^{f}=\left[\begin{array}{c}
-4.2 \\
-0.6 \\
-8.1
\end{array}\right]
$$

Assuming the estimated parameters values for the spherical model the set of consecutive positions for the instantaneous screw axis of the femur with respect to the tibia were calculated. The determined axes intersect at point $A_{3}$. A hodograph is a curve created by the end of the vector with direction of the instantaneous screw axis and magnitude proportional to the angle of rotation about that axis. It is worth noting that angular displacements about consecutive instantaneous screw axes are almost the same. In Fig. 4a paths of points $B_{1}$ and $B_{2}$ were also included.

The consecutive positions of the spherical model calculated at $\alpha \in\left\{10^{\circ} ; 35^{\circ} ; 60^{\circ} ; 85^{\circ}\right\}$ are illustrated in Fig. $4 \mathrm{~b}$. The moving platform is shown only in the first position $\left(\alpha=10^{\circ}\right)$. It is noteworthy that the cruciate ligaments in the spherical model can be seen as intersected in the projection on the sagittal plane $\left(x_{t} y_{t}\right)$ and as skew in space (as in reality).

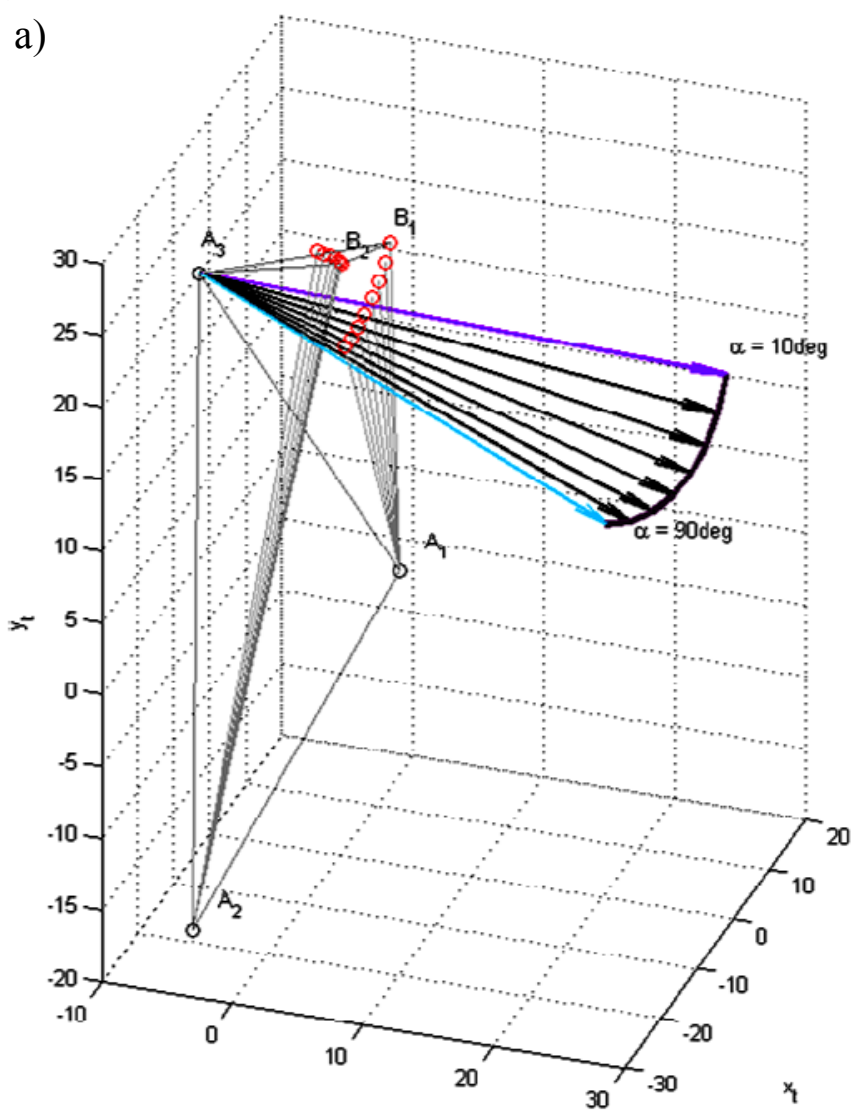

$z_{1}$ b)

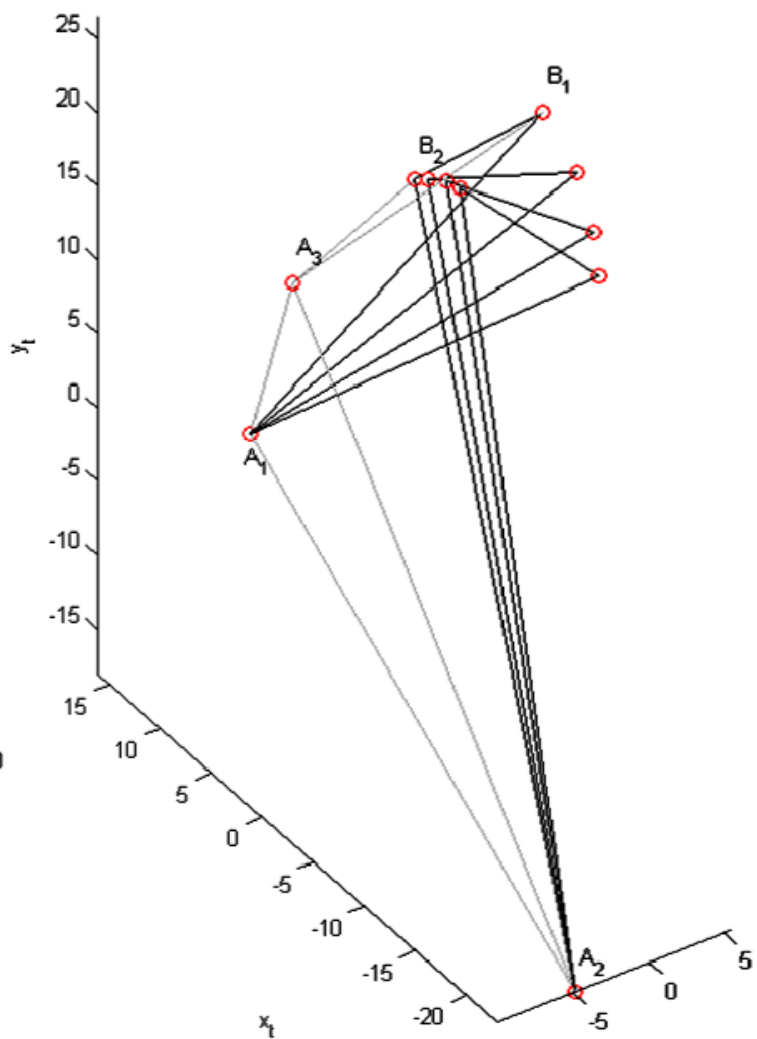

$z_{1}$

Fig.4. a) Hodograph of the vector that has a magnitude proportional to the angular displacement about the instantaneous screw axis in the tibia reference frame. b) Set of several positions of the spherical mechanism in the tibia reference frame. Where: $A_{1} B_{1}, A_{2} B_{2}$ - cruciate ligaments (points $B_{1}$ and $B_{2}$ with caption are calculated at flexion angle $\left.10^{\circ}\right), A_{3}$ - center of the spherical joint connects the platforms, $\alpha-$ flexion/extension angle. 
Functions of displacements obtained from simulation are shown in Fig.5. The resulting set of estimated geometrical parameters allows very good mapping of the knee joint motion in the range of the flexion/extension angle from $10^{\circ}$ to $90^{\circ}$. The greatest incompatibility can be seen in the course of the angle $\beta$. In order to evaluate the usefulness of the sensitivity analysis results obtained from the model with 20 parameters and 18 parameters are presented in graphs in Fig.5. Exclusion of two parameters did not have a noticeable influence on the results.
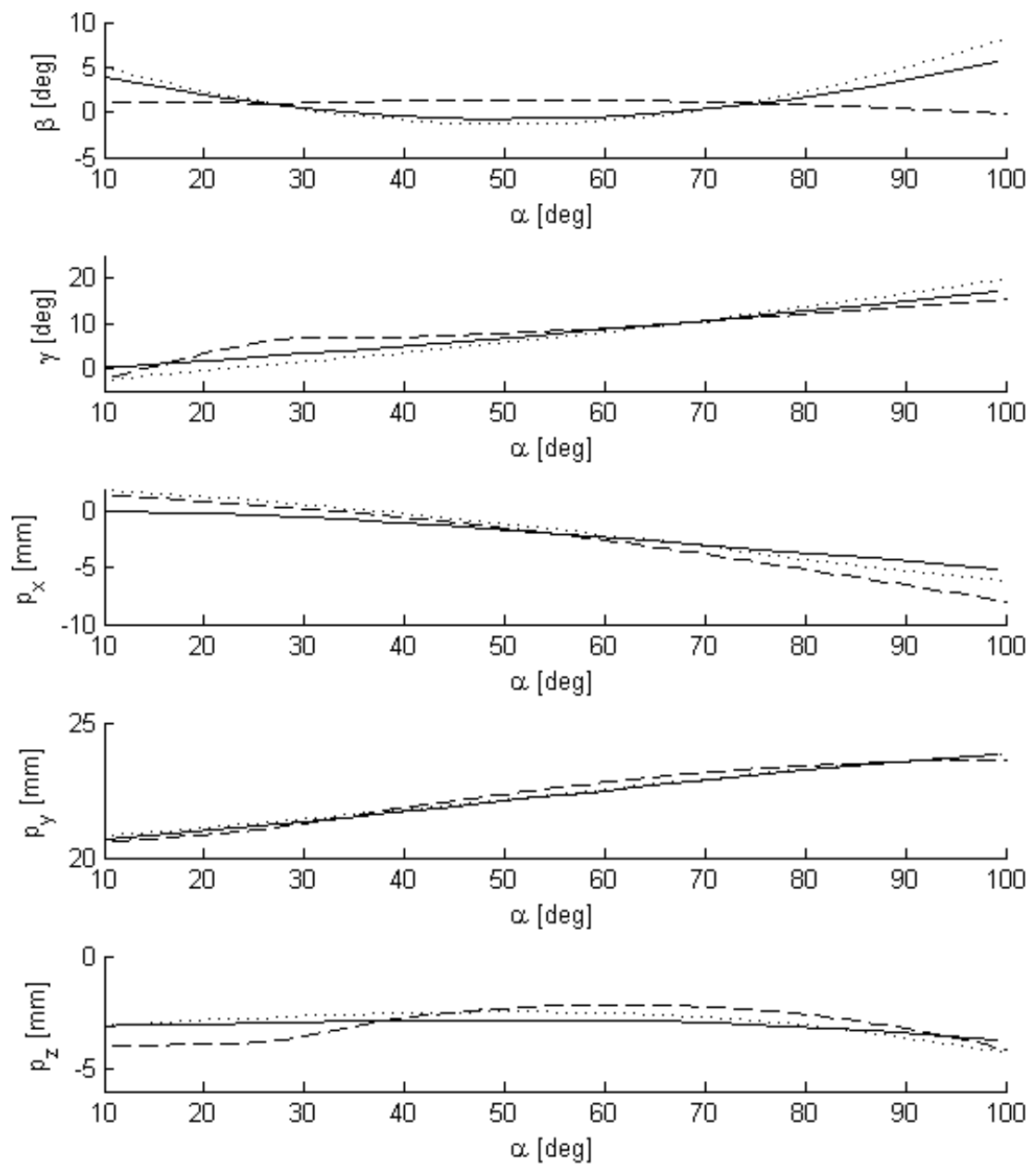

Fig.5. Simulation results: $\beta, \gamma-$ orientation angles, $p_{x}, p_{y}, p_{z}$ - coordinates of the position vector of the femur reference frame in regard to tibia reference frame of the flexion/extension angle $\alpha$, solid line spherical model, dotted line - spherical model with sensitivity analysis, dashed line - spatial model (as per (Sancisi and Parenti-Castelli, 2008)).

\section{Conclusion}

Positions and angular displacements of the spherical model of the knee joint were analysed using the vector method. Sensitivity analysis and then parameters estimation were performed. Numerical simulations proved the effectiveness of the prepared software, the parameters estimation using the vector method and the purpose of sensitivity analysis. This software might be useful in reengineering a damaged joint and designing orthotics for an injured knee as well as a rehabilitation device. 
Simulations were performed for two different data sets obtained through sensitivity analysis and parameters estimation (without sensitivity analysis - spherical model with 20 variable parameters and spherical model with 18 variable parameters and 2 constants). These procedures used a third, experimental data set assumed as per (Sancisi and Parenti-Castelli, 2008) (also called in this paper: input data set). It is important that using different data sets, acquired through parameters estimation, similar results were obtained. Sensitivity analysis helped to simplify the task of parameters estimation.

The vector method was confirmed advantageous in research concerning displacements in the knee joint. It has low numerical complexity because it provides a closed-form solution for the spherical model of the joint.

It seems expedient to use a 3-D scanner to measure the coordinates of the points located on bones on the actual joint and to find a way to perform such experiment as a noninvasive, in vivo test.

\section{Nomenclature}

$\boldsymbol{a}_{i}$ - position vector of the tibia point $A_{i}^{t}$ with respect to the origin of the tibia reference frame $O_{t}$

$\boldsymbol{a}_{i j} \quad$ - vector from point $A_{i}^{t}$ to point $A_{j}^{t}$

$\boldsymbol{b}_{i}$ - position vector of the femur point $B_{i}^{t}$ with respect to the origin of the tibia reference frame $O_{t}$

$\boldsymbol{b}_{i j}-$ vector from point $B_{i}^{t}$ to point $B_{j}^{t}$

$\boldsymbol{b}_{c}-$ position vector of the centroid with respect to point $O_{t}$

$\boldsymbol{d}_{i j} \quad$ - vector from point $B_{i}^{t}\left(A_{i}^{t}\right)$ to point $A_{j}^{t}\left(B_{j}^{t}\right)$

$f$ - objective function used in parameters estimation

$g$ - objective function used to find the $A_{3}$

$l_{j}$ - distance of the selected point from the $j$ instantaneous screw axis

$\boldsymbol{N}$ - matrix used to find the unit quaternion $\boldsymbol{q}$ described with elements $a, b, \ldots, i$ computed using variables $S_{x x}, S_{x y}, \ldots, S_{z z}$

$\boldsymbol{p}$ - translation vector of the origin of the femur reference frame with respect to the tibia reference frame, $\boldsymbol{p}=\left[p_{x} p_{z} p_{y}\right]^{T}$

$\boldsymbol{q}$ - unit quaternion obtained as an eigenvector corresponding to the largest eigenvalue of the matrix $N$

$\boldsymbol{R}$ - rotation matrix of the femur reference frame with respect to tibia reference frame

$r$ - penalty function

$w_{i}-$ weight factor of the respective indicator $(i=1 . .5)$

$x_{r}\left(\alpha_{i}\right)$ - measured value of $x$ at selected $\alpha_{i}$ - flexion angle at position $i$

$x_{s}\left(\alpha_{i}\right)$ - value of $x$ obtained using the spherical model at $\alpha_{i}$-flexion angle at position $i$

$z_{x}$ - range of $x$ obtained from experimental data

$\alpha-$ flexion(+)/extension( -$)$ angle of the femur with respect to the tibia

$\beta-$ abduction( $(-)$ /adduction( + ) angle of the femur with respect to the tibia

$\gamma \quad$ external $(-) /$ internal $(+)$ rotation angle of the femur with respect to the tibia

$\delta$ - input variable in the spherical model - the angle between the first cruciate ligament and the basis (the tibia)

$\sigma$ - influence of the examined, geometrical parameter

$\Delta x \quad$ - indicator of $x \in\left\{\beta, \gamma, p_{x}, p_{y}, p_{z}\right\}$

The following applies to vectors: non-bold symbol stands for the length of the vector, superscript index $o$ stands for the unit vector, superscript index $f$ stands for the femur reference frame (no index means that the vector is described in the tibia reference frame), subscript indices $i$ and $j$ can assume values of $\{1,2,3\}$. 


\section{References}

Blankevoort L., Huiskes R. and De Lange A. (1990): Helical axes of passive knee joint motions. - Journal of Biomechanics, vol.23, No.12, pp.1219-1229.

Fenton R.G. and Shi X. (1990): Comparison of methods for determining screw parameters of finite rigid body motion from initial and final position data. - Trans. ASME, Journal of Mechanical Design, vol.112, pp.472-479.

Grood E.S. and Suntay W.J. (1983): A joint coordinate system for the clinical description of three-dimensional motions: application to the knee. - Trans. ASME, Journal of Biomechanical Engineering, vol.105, No.2, pp.136-144.

Horn B. (1987): Closed-form solution of absolute orientation using quaternions. - Journal of the Optical Society of America A, vol.4, pp.629-642, 1987.

Morecki A., Knapczyk J. and Kędzior K. (2002): Mechanism and Manipulator Theory. - Warsaw: WNT.

Ottoboni A., Parenti-Castelli V., Sancisi N., Belvedere C. and Leardini A. (2010): Articular surface approximation in equivalent spatial parallel mechanism models of the human knee joint: an experiment-based assessment. - Proc. I. Mech. E, Part H: Engineering in Medicine, vol.224, No.9, pp.1121-1132.

Sancisi N. and Parenti-Castelli V. (2010): A 1-Dof parallel spherical wrist for the modelling of the knee passive motion. - Mechanism and Machine Theory, vol.45, No.4, pp.658-665.

Sancisi N. and Parenti-Castelli V. (2008): A new approach for the dynamic modelling of the human knee. - Doctoral thesis, University of Bologna.

Stępniewski A. (2010): Analysis of structural, geometrical and static of knee joint mechanism. - Acta Mechanica et Automatica, vol.4, No.1, pp.80-85.

Received: May 12, 2014

Revised: May 26, 2014 